\title{
GENERALIZED TONIC CLONIC SEIZURES: A RARE CAUSE OF FAHR'S DISEASE IN ADULT
}

Bhawani Shanker Pandey¹, Dharma Rao V², Anitha P3

\section{HOW TO CITE THIS ARTICLE:}

Bhawani Shanker Pandey, Dharma Rao V, Anitha P. "Generalized Tonic Clonic Seizures: A Rare Cause of Fahr's Disease in Adult". Journal of Evolution of Medical and Dental Sciences 2014; Vol. 3, Issue 03, January 20; Page: 695-697, DOI:10.14260/jemds/2014/1888

\begin{abstract}
Fahr's disease is a rare, degenerative, neurological condition characterized by symmetrical and bilateral intracranial calcification. Most cases present with extrapyramidal symptoms. We describe a case of Fahr's disease, who presented with generalized tonic clonic seizure. The diagnosis was made on the basis of combination of clinical features, investigations and exclusion of other causes of intracranial calcification.
\end{abstract}

KEY WORDS: Fahr's disease, generalized tonic clonic seizure.

INTRODUCTION: Fahr's disease is a rare sporadic or familial neurological disorder of unknown etiology characterized by bilateral symmetrical calcification of the basal ganglia, dentate nuclei and cerebellum ${ }^{1}$. This condition has been known since the middle 1800s. The clinical manifestations of Fahr's disease vary. Most cases, therefore, present with extrapyramidal symptoms. One definition proposed by Teautner et al., 1988 requires bilateral calcifications with neuropsychiatric and extrapyramidal disorders with normal calcium and phosphorus metabolism. Bealle et al., 1989 gave another definition which had seizures, rigidity, and dementia with characteristic calcification of the basal ganglia. Here we report a case who presented with generalized tonic clonic seizure which is an uncommon presentation. At the present time, CT scan remains the most cost effective screening tool for Fahr's disease ${ }^{2}$.

CASE REPORT: A 21 year old female patient was brought to emergency department with history of one episode of generalized tonic clonic seizure at home. She had one more episode of seizure in the emergency department. There was no recent history of headache, vomiting, fever and head injury. Past history from parents revealed history of febrile seizures at the age of two years and she was on antiepileptic drugs for 4 years but documents were not available. She was seizure free till now. Family history revealed that mother and younger brother also had seizure disorder.

Parents had noticed abnormal behavior like not taking food, excessive water drinking, burst of inappropriate and uncontrollable laughing, crying spells, suicidal thoughts, passing of urine in clothes for the last 3 years. No involuntary movements were noticed. She was under psychiatrist care for paranoid schizophrenia before the present complaints.

On examination, patient was drowsy. Her GCS was $E_{2} V_{3} M_{5}$; pulse rate 100/min, regular; Blood pressure 90/60 mmHg. Pupils were of normal size and well reacting to light. No focal neurological deficit was detected. Standard protocol for seizures management was initiated. Laboratory studies CBP, CUE, RBS, Blood urea, Serum creatinine, Serum electrolytes, Serum calcium, Serum magnesium, Serum phosphate, TSH, Liver function tests were normal. Her CECT brain showed bilateral symmetrical extensive calcifications in basal ganglia, thalamus, centrum semiovale, cerebellar hemispheres (Fig 1). Brain CT Scan of her brother and mother were normal. Patient 
improved over one week. Patient was discharged after prescribing antiepileptic and antipsychotic medication.

DISCUSSION: A plethora of descriptive terms have been used to describe this disease, such as symmetric cerebral calcification, idiopathic calcification of cerebral capillaries, symmetrical calcification of basal ganglia, idiopathic familial cerebrovascular ferrocalcinosis, bilateral striatopallido dentate calcinosis and known with a total of 35 names resulting in considerable confusion as to what cases constitute Fahr's disease. Delacour first described calcification of the basal ganglia in $1850^{3}$. Bamberger described the histopathological entity of calcification of the fine cerebral vessels in 18553. Fahr described a case report of a man who possibly had Hypoparathyroidism and autopsy revealed calcification in the white matter with little calcification of basal ganglia.

Bala V Manyam et al combined the literature and the Fahr's disease registry sources and found that the most common manifestations of Fahr's disease was movement disorders (55\%) ${ }^{2}$. Parkinsonism accounted for over half of all movement disorders (57\%), while hyperkinetic movement disorders accounted for the rest. Cognitive impairment was the second most common manifestation followed by cerebellar impairment and speech disorder. Overlap of neurologic manifestations such as hypokinetic movement disorders associated with cognitive impairment and cerebellar signs were often present.

Typically the age at onset of clinical symptoms is 30 to 60 years. The clinical evolution is that of a degenerative disorder, rather than a developmental disorder. Fahr's disease may present in familial form suggestive of psychosis and generalized tonic clonic seizures with intellectual decline ${ }^{4}$. There was no evidence of hypocalcaemia and hyperphosphatemia in our patient. We screened the family members of the patient for possible asymptomatic state of the disease but none of them had any evidence of intracranial calcification.

Clinical diagnosis is facilitated by the presence of bilateral and symmetrical calcifications in the basal ganglia in cranial CT. In suspected cases, other differential causes of intracranial calcification must be ruled out, such as parathyroid disorders, vascular lesions, and infectious diseases like toxoplasmosis, syphilis and inflammatory illnesses such as systemic lupus erythematosus. CT scan remains the most effective screening tool among the brain imaging techniques. It helps in detection of site, extent and volume of involvement of calcification. Detection of intracranial calcifications in CT scan is more sensitive comparing with skull X-ray or MRI. In the present case, clinical diagnosis of Fahr's disease was done on the combination of clinical features, brain imaging and exclusion of other causes of intracranial calcification ${ }^{5}$. Clinical presentations may be quite variable. Neurological and psychiatric symptoms, if present at all, are highly variable and include progressive mental deterioration, convulsive seizures, parkinsonism, dysarthria, ataxia, psychosis or affective disorders.

There is neither a cure nor a standard course of treatment. Treatment addresses symptoms on an individual basis. Correction of calcium and phosphate levels may lead to clinical improvement, in particular, disappearance of epileptic seizures and abnormal movements. The use of antipsychotic may be indicated in those presenting with psychotic symptoms or behavioral problems. The choice of atypical antipsychotics or those with less extra-pyramidal side-effects is warranted because of the 
co-existence of extra-pyramidal symptoms in this group of patients. The prognosis is variable and hard to predict.

CONCLUSION: The present case emphasizes that cases presenting with seizure symptomatology must be thoroughly investigated; family history and screening be given due importance if associated with cognitive and behavioral abnormalities and possibility of disorders presenting with protean clinical manifestations as Fahr's disease be considered.

\section{REFERENCE:}

1. Shamim VS, Joel PT, James AB. Paranoid Delusions and cognitive impairment suggesting Fahr's disease, Psychosomatic, 2005; 45: 569-572.

2. Bala V. Manyam. What is and what is not 'Fahr's disease': Parkinsonism and related disorders 2005; 11: 73-80.

3. Morgante L, Trimarchi F, Benvenga S. Fahr's disease. The Lancet 2002;359:759

4. S. Srivastava, Manjeet S. Bhatia, V. Sharma, S Mahajan, G Rajendar. Fahr's disease: an incidental finding in a case presenting with psychosis. German Journal of Psychiatry. 2010; 13 (2): 86-90.

5. Joyce SPH, Samson YYF, Yin GC, Wing YK. Fahr's disease: a differential diagnosis of frontal lobe syndrome; Hong Kong Med J 2007; 13: 75-7.

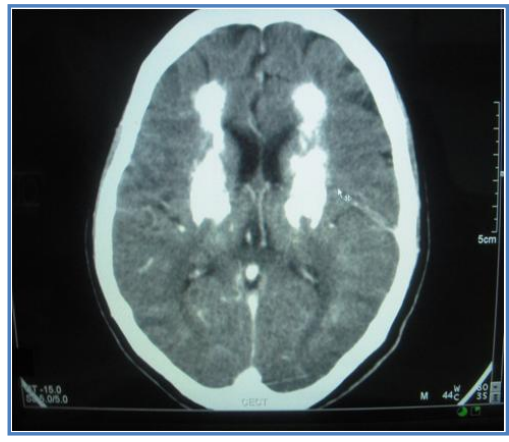

\section{Fig. 1: Calcification in the Basal Ganglia, Thalamus, and Cerebral cortex}

\section{AUTHORS:}

1. Bhawani Shanker Pandey

2. Dharma Rao V.

3. Anitha P.

\section{PARTICULARS OF CONTRIBUTORS:}

1. Assistant Professor, Department of Internal Medicine, Mamata Medical College, Khammam.

2. Professor, Department of Internal Medicine, Mamata Medical College, Khammam.

3. Civil Surgeon Specialist, Department of Internal Medicine, ESI Hospital, Hyderabad.
NAME ADDRESS EMAIL ID OF THE CORRESPONDING AUTHOR:
Dr. Dharma Rao V., Block No. 7, Kinnerasani, Staff Qtrs, Mamata General Hospital, Khammam, Andhra Pradesh, PIN - 507002.
Email - vdrao1@rediffmail.com
Date of Submission: 19/12/2013.
Date of Peer Review: 20/12/2013.
Date of Acceptance: 05/01/2014.
Date of Publishing: 16/01/2014. 